2018

\title{
Axioms for the fixed point index of n-valued maps, and some applications
}

Christopher P. Staecker

Fairfield University, cstaecker@fairfield.edu

Follow this and additional works at: https://digitalcommons.fairfield.edu/mathandcomputerscience-

facultypubs

Copyright 2018 Springer-Verlag

"This is a post-peer-review, pre-copyedit version of an article published in Journal of Fixed Point Theory and Applications. The final authenticated version is available online at: http://dx.doi.org/ 10.1007/s11784-018-0543-4".

The author post-print has been archived here with permission from the copyright holder.

\section{Peer Reviewed}

\section{Repository Citation}

Staecker, Christopher P., "Axioms for the fixed point index of n-valued maps, and some applications" (2018). Mathematics Faculty Publications. 51.

https://digitalcommons.fairfield.edu/mathandcomputerscience-facultypubs/51

\section{Published Citation}

Staecker, P. C. (2018). Axioms for the fixed point index of n-valued maps, and some applications. Journal of Fixed Point Theory and Applications, 20(61). doi:10.1007/s11784-018-0543-4.

This Article is brought to you for free and open access by the Mathematics Department at DigitalCommons@Fairfield. It has been accepted for inclusion in Mathematics Faculty Publications by an authorized administrator of DigitalCommons@Fairfield. For more information, please contact digitalcommons@fairfield.edu. 


\title{
Axioms for the fixed point index of $n$-valued maps, and some applications
}

\author{
P. Christopher Staecker
}

February 17, 2018

\begin{abstract}
We give an axiomatic characterization of the fixed point index of an $n$-valued map. For $n$-valued maps on a polyhedron, the fixed point index is shown to be unique with respect to axioms of homotopy invariance, additivity, and a splitting property. This uniqueness is used to obtain easy proofs of an averaging formula and product formula for the index. In the setting of $n$-valued maps on a manifold, we show that the axioms can be weakened.
\end{abstract}

\section{Introduction}

Given a set $X$ and a positive integer $n$, an $n$-valued function is a set-valued function $f$ on $X$ such that $f(x)$ has cardinality exactly $n$ for every $x \in X$. Equivalently, an $n$-valued function on $X$ is a single-valued function $f: X \rightarrow$ $D_{n}(Y)$, where $D_{n}(Y)$ is the unordered configuration space of $n$ points in $Y$, defined as:

$$
D_{n}(Y)=\left\{\left\{y_{1}, \ldots, y_{n}\right\} \mid y_{i} \in Y, y_{i} \neq y_{j} \text { for } i \neq j\right\} .
$$

When $Y$ is a topological space, we give $D_{n}(Y)$ a topology as follows: begin with the product topology on $Y^{n}$, then consider the subspace of tuples $\left(y_{1}, \ldots, y_{n}\right) \in Y^{n}$ with $y_{i} \neq y_{j}$ for $i \neq j$. Then $D_{n}(Y)$ is the quotient of these tuples up to ordering, and so its topology is given by the quotient topology. When $f: X \rightarrow D_{n}(Y)$ is continuous, we call it an $n$-valued map from $X$ to $Y$.

Continuity of $n$-valued maps can also be defined in terms of lower- and upper-semicontinuity. These approaches are equivalent: see [?].

We say that an $n$-valued map $f: X \rightarrow D_{n}(Y)$ is split if there are $n$ single valued continuous functions $f_{1}, \ldots, f_{n}: X \rightarrow Y$ with $f(x)=\left\{f_{1}(x), \ldots, f_{n}(x)\right\}$ for every $x \in X$. In this case we write $f=\left\{f_{1}, \ldots, f_{n}\right\}$. Not all $n$-valued maps are split.

In this paper all spaces will be assumed to be finite polyhedra, and we will generally focus on $n$-valued selfmaps of the form $f: X \rightarrow D_{n}(X)$. A fixed point of such a map is a point $x \in X$ with $x \in f(x)$. 
Topological fixed point theory of $n$-valued maps was first studied by Schirmer in $[?, ?]$. Schirmer's approach is fundamentally based on the following lemma of Banach and Mazur [?]:

Lemma 1 (Splitting Lemma). If $X$ is simply connected, then any $n$-valued map $f: X \rightarrow D_{n}(Y)$ is split.

The local fixed point index is a classical invariant of a self-map which gives an algebraic count of the "multiplicity" of a fixed point. We will write the classical local fixed point index of a single-valued map $f$ on the set $U$ as $\operatorname{ind}(f, U)$.

The index is typically defined geometrically or homologically, see [?]. There is also a substantial literature taking an axiomatic approach. The fixed point index is the unique numerical homotopy invariant which satisfies certain properties. In [?], in the context of manifolds, it is shown that the index is unique with respect to axioms of homotopy, additivity, and a normalization for constant maps. This approach has been adapted to similar results for the coincidence index [?, ?] and the Reidemeister trace [?]. Other axiom schemes have been used for the Lefshetz number, see [?].

Schirmer in [?] defines the local fixed point index of an $n$-valued map. For clarity we write this index, when $f$ is an $n$-valued map, as $\operatorname{ind}_{n}(f, U)$. Schirmer's definition is as follows: First change $f$ by homotopy to some map $f^{\prime}$ which has finitely many isolated fixed points $x_{j}$ inside $U$. Then choose a simply connected neighborhood $V_{j} \subset U$ around each fixed point. Then $f$ splits on $V_{j}$ as $f=$ $\left\{f_{1}, \ldots, f_{n}\right\}$, and $x_{j}$ will be a fixed point of exactly one of the $f_{i}$. Shirmer then defines $\operatorname{ind}_{n}(f, U)=\sum_{j} \operatorname{ind}\left(f_{i}, V_{j}\right)$, where ind denotes the classical fixed point index of a single-valued map. Schirmer proves that the index is well defined and is homotopy invariant and additive on disjoint subsets.

The fixed point index of an $n$-valued map is also discussed by Crabb [?]. Crabb in particular demonstrates an $n$-valued form of the Lefschetz-Hopf Theorem, that the fixed point index of $(f, X)$ equals the Lefschetz number, the alternating sum of the traces of some induced map in homology. The Lefschetz number of an $n$-valued map was defined by Brown in [?]. If we denote the Lefschetz number of some $n$-valued map as $L(f)$, Proposition 3.5 and Corollary 5.9 of [?] give:

Theorem 2. (Crabb, 2015) Let $f: X \rightarrow D_{n}(X)$ be an $n$-valued map. Then

$$
L(f)=\operatorname{ind}(f, X) .
$$

In Section ?? we prove a uniqueness theorem for the fixed point index of an $n$-valued map on a polyhedron. We show that $\operatorname{ind}_{n}$ is the unique function satisfying homotopy invariance, additivity on disjoint subsets, and a splitting property. As applications of our uniqueness theorem we prove in Sections ?? and ?? an averaging and product formula for the index. (The product formula also appears in Proposition 3.4 of [?].) In Section ?? we prove a stronger uniqueness theorem (using weaker axioms) in the setting of $n$-valued maps on manifolds.

We would like to thank Michael Crabb for bringing the work in [?] to our attention. 


\section{The axioms}

In this section we prove a uniqueness theorem for the local fixed point index of an $n$-valued map using axioms inspired by [?]. That paper uses the setting of maps on differentiable manifolds, but our strong third axiom will allow the more general setting of maps on any complex.

Given a complex $X$, we say a pair $(f, U)$ is admissible when $f$ is an $n$-valued selfmap on $X$ with $U \subset X$ and $\operatorname{Fix}(f) \cap U$ is compact. (Schirmer in [?] defines $(f, U)$ as admissible when $\operatorname{Fix}(f) \cap \partial U=\emptyset$. The present definition is equivalent since $\operatorname{Fix}(f)$ is a closed set and the complex $X$ is compact.) A homotopy $H$ : $X \times[0,1] \rightarrow X$ of some admissible pair $(f, U)$ to $(g, U)$ is admissible when $\{(x, t) \mid x \in H(x, t) \cap U\}$ is compact.

For some complex $X$, let $\mathcal{C}_{n}(X)$ be the set of admissible pairs $(f, U)$ where $f$ is an $n$-valued selfmap on $X$. We will write $\mathcal{C}(X)=\mathcal{C}_{1}(X)$ for the single-valued case. In the following, let $\iota$ be a real-valued function $\iota: \mathcal{C}_{n}(X) \rightarrow \mathbb{R}$.

Axiom 3. (Homotopy) Let $(f, U),(g, U) \in \mathcal{C}_{n}(X)$ be admissibly homotopic. Then $\iota(f, U)=\iota(g, U)$.

Axiom 4. (Additivity) Let $(f, U) \in \mathcal{C}_{n}(X)$ with $\operatorname{Fix}(f) \cap U \subseteq U_{1} \sqcup U_{2}$, where $\sqcup$ denotes disjoint union. Then $\iota(f, U)=\iota\left(f, U_{1}\right)+\iota\left(f, U_{2}\right)$.

Axiom 5. (Splitting) Let $(f, U) \in \mathcal{C}_{n}(X)$ such that on the set $U$, the map $f$ splits as $\left\{f_{1}, \ldots, f_{n}\right\}$, and $\operatorname{Fix}(f) \cap U$ is a single point. Then

$$
\iota(f, U)=\operatorname{ind}\left(f_{i}, U\right),
$$

where $f_{i}$ is the unique map with $\operatorname{Fix}\left(f_{i}\right) \cap U \neq \emptyset$.

Schirmer proves each of these axioms are satisfied when $\iota$ is the local fixed point index of an $n$-valued map. The homotopy axiom is Theorem 4.5 of [?], the additivity axiom is Theorem 4.6 of [?], and the splitting axiom is an immediate consequence of the definition of the index, which is defined in terms of splittings.

An immediate consequence of the additivity axiom is the following property:

Theorem 6. [Excision property] Let $\iota$ satisfy the additivity axiom. If $(f, U) \in$ $\mathcal{C}_{n}(X)$ and $V \subset U$ with $(f, V) \in \mathcal{C}_{n}(X)$ and $\operatorname{Fix}(f) \cap U \subseteq V$, then $\iota(f, U)=$ $\iota(f, V)$.

Proof. First we prove an "empty set property", that $\iota(f, \emptyset)=0$. This is proved by writing the disjoint union $\operatorname{Fix}(f) \cap \emptyset \subseteq \emptyset \sqcup \emptyset$, and so

$$
\iota(f, \emptyset)=\iota(f, \emptyset)+\iota(f, \emptyset)
$$

and thus $\iota(f, \emptyset)=0$.

No we prove the excision property. Writing $\operatorname{Fix}(f) \cap U \subseteq \emptyset \sqcup V$ and applying the additivity axiom gives:

$$
\iota(f, U)=\iota(f, \emptyset)+\iota(f, V)=\iota(f, V) .
$$


In the case when $\operatorname{Fix}(f) \cap U$ is empty, the excision property gives $\iota(f, U)=$ $\iota(f, \emptyset)=0$. Thus we obtain:

Theorem 7. [Solution property] Let $\iota$ satisfy the additivity axiom. If $(f, U) \in$ $\mathcal{C}_{n}(X)$ and $\iota(f, U) \neq 0$, then $\operatorname{Fix}(f) \cap U$ is nonempty.

Recall Schirmer's definition of the index: starting with an admissible pair $(f, U)$, first we replace $f$ by an admissibly homotopic map $f^{\prime}$ so that $\operatorname{Fix}\left(f^{\prime}\right) \cap U$ is finite. The existence of such a "fix-finite" homotopy is the main result of [?]. Then we choose simply connected isolating neighborhoods $V_{j}$ of the fixed points of $f^{\prime}$ in $U$. Since $V_{j}$ are simply connected we have a splitting $f=\left\{f_{1}, \ldots, f_{n}\right\}$ on $V_{j}$, and there is some $i$ such that $\operatorname{Fix}(f) \cap V_{j}=\operatorname{Fix}\left(f_{i}\right) \cap V_{j}$ is a single point. Then Schirmer defines:

$$
\operatorname{ind}_{n}(f, U)=\sum_{j} \operatorname{ind}\left(f_{i}, V_{j}\right) .
$$

Schirmer proves that this definition is independent of the various choices of $f^{\prime}$ and the sets $V_{j}$. But we remark that Schirmer's definition of ind ${ }_{n}$ is constructed entirely using the properties of the Homotopy, Additivity, and Splitting axioms. That is, if $\iota$ is any function satisfying these three axioms, then following the same construction above we must also have

$$
\iota(f, U)=\sum_{j} \operatorname{ind}\left(f_{i}, V_{j}\right) .
$$

Thus we immediately obtain:

Theorem 8. Given any complex $X$, the local fixed point index is the unique function $\iota: \mathcal{C}_{n}(X) \rightarrow \mathbb{R}$ obeying the Homotopy, Additivity, and Splitting axioms.

Note that $\operatorname{ind}_{n}$ is proved to be the unique real-valued function satisfying the axioms. In fact the values of the fixed point index are always integers.

\section{$3 \quad$ An averaging formula}

In this section we obtain an $n$-valued analogue of the averaging formula for the fixed point index. The first averaging formula of this kind is apparently Jiang's averaging formula for the Lefschetz number which appears as Theorem III.2.12 of [?]. When $f: X \rightarrow X$ is a single-valued selfmap and $p: \bar{X} \rightarrow X$ is a finite covering with covering group $G$, let $\bar{f}: \bar{X} \rightarrow \bar{X}$ be a lift of $f$. Then the averaging formula states:

$$
L(f)=\frac{1}{|G|} \sum_{\alpha \in G} L(\alpha \bar{f}) .
$$

This is a special case of an averaging formula for the local fixed point index, which is straightforward but does not seem to have appeared in the literature until recently. An axiomatic proof is given in [?], as well as a generalization to the coincidence Reidemeister trace. 
Theorem 9. Let $f: X \rightarrow X$ be a selfmap and $p: \bar{X} \rightarrow X$ is a finite covering with covering group $G$, let $\bar{f}: \bar{X} \rightarrow \bar{X}$ be a lift of $f$. If $(f, U) \in \mathcal{C}(X)$, then $\left(\bar{f}, p^{-1}(U)\right) \in \mathcal{C}(\bar{X})$ and

$$
\operatorname{ind}(f, U)=\frac{1}{|G|} \sum_{\alpha \in G} \operatorname{ind}\left(\alpha \bar{f}, p^{-1}(U)\right) .
$$

Our goal for this section is to prove a similar formula for ind ${ }_{n}$.

Given a single valued map $g: X \rightarrow Y$, let $g^{n}$ be the function which applies $g$ to each element of an $n$-element set. (This function $g^{n}$ may not always be $n$-valued.)

When $\bar{X} \rightarrow X$ is a covering map, and $f: X \rightarrow D_{n}(X)$ is an $n$-valued map, say that an $n$-valued map $\bar{f}: \bar{X} \rightarrow D_{n}(\bar{X})$ is a lifting of $f$ when the following diagram commutes:

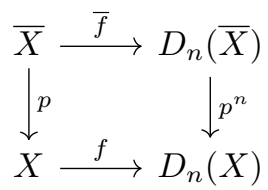

Let $G$ be the group of covering transformations for the covering $p: \bar{X} \rightarrow X$, and $\bar{f}: \bar{X} \rightarrow D_{n}(\bar{X})$ be a lift of some $n$-valued map $f: X \rightarrow D_{n}(X)$. Viewing any $\alpha \in G$ as an automorphism of $\bar{X}$, the composition $\alpha^{n} \bar{f}: \bar{X} \rightarrow D_{n}(\bar{X})$ is always an $n$-valued map, which is another lift of $f$.

Our averaging formula takes the following form:

Theorem 10. Let $p: \bar{X} \rightarrow X$ be a finite covering map, and let $G$ be the group of covering transformations. Let $f: X \rightarrow D_{n}(X)$ be an n-valued map, and let $\bar{f}: \bar{X} \rightarrow D_{n}(\bar{X})$ be a lifting. Let $(f, U) \in \mathcal{C}_{n}(X)$, and let $\bar{U}=p^{-1}(U)$. Then $\left(\alpha^{n} \bar{f}, \bar{U}\right) \in \mathcal{C}_{n}(\bar{X})$ for each $\alpha \in G$, and

$$
\operatorname{ind}_{n}(f, U)=\frac{1}{|G|} \sum_{\alpha \in G} \operatorname{ind}_{n}\left(\alpha^{n} \bar{f}, \bar{U}\right) .
$$

Proof. Since $(f, U) \in \mathcal{C}_{n}(X)$ and $\bar{X}$ is a finite cover of $X$, it is clear that $(\bar{f}, \bar{U}) \in$ $\mathcal{C}_{n}(\bar{X})$, and thus that $\left(\alpha^{n} \bar{f}, \bar{U}\right) \in \mathcal{C}_{n}(\bar{X})$, since $\alpha^{n}: D_{n}(\bar{X}) \rightarrow D_{n}(\bar{X})$ is a homeomorphism.

Given an admissible pair $(f, U)$, let $\iota$ be defined by:

$$
\iota(f, U)=\frac{1}{|G|} \sum_{\alpha \in G} \operatorname{ind}_{n}\left(\alpha^{n} \bar{f}, \bar{U}\right) .
$$

We must show that $\iota(f, U)=\operatorname{ind}_{n}(f, U)$, and we do this by showing that $\iota$ satisfies the Homotopy, Additivity, and Splitting axioms.

For the Homotopy axiom, let $\left(f^{\prime}, U\right)$ be admissibly homotopic to $(f, U)$. Then the admissible homotopy from $(f, U)$ to $\left(f^{\prime}, U\right)$ naturally lifts to an admissible homotopy starting at $\left(\alpha^{n} \bar{f}, \bar{U}\right)$ and ending with $\left(\alpha^{n} \bar{f}^{\prime}, \bar{U}\right)$, where $\bar{f}^{\prime}$ is 
some lifting of $f^{\prime}$. Then by the homotopy property of ind $\mathrm{d}_{n}$ we have:

$$
\iota(f, U)=\frac{1}{|G|} \sum_{\alpha \in G} \operatorname{ind}_{n}\left(\alpha^{n} \bar{f}, \bar{U}\right)=\frac{1}{|G|} \sum_{\alpha \in G} \operatorname{ind}_{n}\left(\alpha^{n} \bar{f}^{\prime}, \bar{U}\right)=\iota\left(f^{\prime}, U\right)
$$

and so $\iota$ satisfies the homotopy axiom.

For the Additivity axiom, let $\operatorname{Fix}(f) \cap U \subseteq U_{1} \sqcup U_{2}$. Then let $\bar{U}_{i}=p^{-1}\left(U_{i}\right)$ for $i=1,2$, and we have $\operatorname{Fix}\left(\alpha^{n} \bar{f}\right) \cap \bar{U} \subseteq \bar{U}_{1} \sqcup \bar{U}_{2}$. Thus the additivity property of $\operatorname{ind}_{n}$ gives:

$$
\begin{aligned}
\iota(f, U) & =\frac{1}{|G|} \sum_{\alpha \in G} \operatorname{ind}_{n}\left(\alpha^{n} \bar{f}, \bar{U}\right) \\
& =\frac{1}{|G|} \sum_{\alpha \in G}\left(\operatorname{ind}_{n}\left(\alpha^{n} \bar{f}, \bar{U}_{1}\right)+\operatorname{ind}_{n}\left(\alpha^{n} \bar{f}, \bar{U}_{2}\right)\right) \\
& =\iota\left(f, U_{1}\right)+\iota\left(f, U_{2}\right) .
\end{aligned}
$$

For the Splitting axiom, say that $f$ splits on $U$ as $\left\{f_{1}, \ldots, f_{n}\right\}$, and $\operatorname{Fix}(f) \cap$ $U=\operatorname{Fix}\left(f_{i}\right) \cap U$ is a single point. Lifting the maps $f_{i}$, we obtain a splitting of $\bar{f}$ as $\bar{f}=\left\{\bar{f}_{1}, \ldots, \bar{f}_{n}\right\}$. First we claim that, for each $\alpha \in G$, we have $\operatorname{ind}_{n}\left(\alpha^{n} \bar{f}, \bar{U}\right)=$ $\operatorname{ind}_{n}\left(\alpha \underline{f}_{i}, \bar{U}\right)$. In the case where $\operatorname{Fix}(\alpha \bar{f}) \cap \bar{U}=\emptyset$, then $\operatorname{ind}_{n}\left(\alpha^{n} \bar{f}, \bar{U}\right)=0=$ $\operatorname{ind}_{n}\left(\alpha \bar{f}_{i}, \bar{U}\right)$ as desired. In the case where $\operatorname{Fix}\left(\alpha^{n} \bar{f}\right) \cap \bar{U}$ is not empty, note that when $j \neq i$ then $\operatorname{Fix}\left(\alpha^{n} \bar{f}_{j}\right) \cap \bar{U}=\emptyset$, since otherwise $f_{j}$ would have a fixed point in $U$. Thus $\operatorname{Fix}\left(\alpha \bar{f}_{i}\right) \cap \bar{U}$ must be nonempty, and the splitting property of $\operatorname{ind}_{n}$ $\operatorname{gives}_{\operatorname{ind}_{n}}\left(\alpha^{n} \bar{f}, \bar{U}\right)=\operatorname{ind}\left(\alpha \bar{f}_{i}, \bar{U}\right)$.

Thus for each $\alpha$ we have $\operatorname{ind}_{n}\left(\alpha^{n} \bar{f}, \bar{U}\right)=\operatorname{ind}\left(\alpha \bar{f}_{i}, \bar{U}\right)$, and so:

$$
\iota(f, U)=\frac{1}{|G|} \sum_{\alpha \in G} \operatorname{ind}_{n}\left(\alpha^{n} \bar{f}, \bar{U}\right)=\frac{1}{|G|} \sum_{\alpha \in G} \operatorname{ind}\left(\alpha \bar{f}_{i}, \bar{U}\right)=\operatorname{ind}\left(f_{i}, U\right)
$$

where the last equality is the averaging formula for the classical fixed point index.

Taking $U=X$ in the theorem above, we obtain an averaging formula for the Lefschetz number of an $n$-valued map:

Corollary 11. Let $p: \bar{X} \rightarrow X$ be a finite covering map, and let $G$ be the group of covering transformations. Let $f: X \rightarrow D_{n}(X)$ be an $n$-valued map, and let $\bar{f}: \bar{X} \rightarrow D_{n}(\bar{X})$ be a lifting. Then:

$$
L(f)=\frac{1}{|G|} \sum_{\alpha \in G} L\left(\alpha^{n} \bar{f}\right) .
$$

\section{A product formula}

In this section we prove a product formula for the index of an $n$-valued map, again using the axioms of Theorem ??. 
Our aim is to generalize the well known product formula for the index of a single valued map (see [?], I.3.5.iv):

$$
\operatorname{ind}(f \times g, U \times V)=\operatorname{ind}(f, U) \operatorname{ind}(g, V) .
$$

Given an $n$-valued map $f: X \rightarrow D_{n}(X)$ and an $m$-valued map $g: Y \rightarrow$ $D_{m}(Y)$, the product $f \times g: X \times Y \rightarrow D_{m n}(X \times Y)$ is the $m n$-valued map given by:

$$
(f \times g)(x, y)=f(x) \times g(y) .
$$

The goal for this section is to prove the following product formula:

$$
\operatorname{ind}_{m n}(f \times g, U \times V)=\operatorname{ind}_{n}(f, U) \operatorname{ind}_{m}(g, V) .
$$

This formula appears as part of Proposition 3.4 of [?], where it is proved using entirely different methods.

It is not clear exactly how to approach the product formula by directly using Theorem ??. Instead we prove a simple variation of Theorem ?? specialized to the category of product mappings.

Given complexes $X$ and $Y$ and positive integers $n$ and $m$, let $\mathcal{P}_{n, m}(X, Y)$ be the set of all pairs $(f \times g, U \times V)$ where $f: X \rightarrow D_{n}(X)$ is an $n$-valued map and $g: Y \rightarrow D_{n}(Y)$ is an $m$-valued map, and $(f, U) \in \mathcal{C}_{n}(X)$ and $(g, V) \in \mathcal{C}_{m}(Y)$.

Theorem 12. The local fixed point index $\operatorname{ind}_{m n}(f, U)$ for mn-valued maps is the unique real-valued function $\iota: \mathcal{P}_{n, m}(X, Y) \rightarrow \mathbb{R}$ satisfying the following axioms:

- (Product Homotopy) Let $(f, U)$ be admissibly homotopic to $\left(f^{\prime}, U\right)$ and $(g, V)$ be admissibly homotopic to $\left(g^{\prime}, V\right)$. Then $\iota(f \times g, U \times V)=\iota\left(f^{\prime} \times\right.$ $\left.g^{\prime}, U \times V\right)$.

- (Additivity) Let $(f \times g, U \times V) \in \mathcal{P}_{n, m}(X, Y)$ with $\operatorname{Fix}(f \times g) \cap(U \times V) \subset$ $\left(U_{1} \times V_{1}\right) \sqcup\left(U_{2} \times V_{2}\right)$. Then $\iota(f \times g, U \times V)=\iota\left(f \times g, U_{1} \times V_{1}\right)+\iota(f \times$ $\left.g, U_{2} \times V_{2}\right)$.

- (Splitting) Let $(f \times g, U \times V) \in \mathcal{P}_{n, m}(X, Y)$ such that $f \times g$ splits on $U \times V$ as $\left(h_{1}, \ldots, h_{m n}\right)$, and $\operatorname{Fix}(f \times g) \cap U \times V=\operatorname{Fix}\left(h_{i}, U \times V\right)$ is a single point. Then

$$
\iota(f \times g, U \times V)=\operatorname{ind}\left(h_{i}, U \times V\right) .
$$

Proof. Let $\iota: \mathcal{P}_{n, m}(X, Y) \rightarrow \mathbb{R}$ satisfy the three axioms, and let $(f \times g, U \times V) \in$ $\mathcal{P}_{n, m}(X, Y)$. We will show that $\iota(f \times g, U \times V)=\operatorname{ind}_{m n}(f \times g, U \times V)$.

Schirmer's fix-finite theorem of [?] applied to $f$ and $g$ provides admissible pairs $\left(f^{\prime}, U\right)$ and $\left(g^{\prime}, V\right)$ such that $(f, U)$ is admissibly homotopic to $\left(f^{\prime}, U\right)$ and $(g, V)$ is admissibly homotopic to $\left(g^{\prime}, V\right)$ and $\operatorname{Fix}\left(f^{\prime}\right) \cap U$ and $\operatorname{Fix}\left(g^{\prime}\right) \cap V$ are finite sets. By the product homotopy axiom, we have

$$
\iota(f \times g, U \times V)=\iota\left(f^{\prime} \times g^{\prime}, U \times V\right) .
$$


It is clear to see that $\operatorname{Fix}\left(f^{\prime} \times g^{\prime}\right) \cap(U \times V)=\left(\operatorname{Fix}\left(f^{\prime}\right) \cap U\right) \times\left(\operatorname{Fix}\left(g^{\prime}\right) \cap V\right)$, and in fact if $U_{1}, \ldots, U_{k} \subset U$ are isolating neighborhoods of the fixed points of $f^{\prime}$, and $V_{1}, \ldots, V_{l} \subset V$ are isolating neighborhoods of the fixed points of $g^{\prime}$, then the various $U_{i} \times V_{j}$ are isolating neighborhoods of the fixed points of $f^{\prime} \times g^{\prime}$, and thus the additivity axiom gives:

$$
\iota(f \times g, U \times V)=\sum_{s=1}^{k} \sum_{t=1}^{l} \iota\left(f^{\prime} \times g^{\prime}, U_{s} \times V_{t}\right) .
$$

We may assume that the $U_{s}$ and $V_{t}$ are all simply connected, and so $U_{s} \times V_{t}$ is simply connected, and so $f^{\prime} \times g^{\prime}$ splits on each $U_{s} \times V_{t}$ as $\left(h_{1, s, t}, \ldots, h_{m n, s, t}\right)$, and there is exactly one value of $i$ such that $\operatorname{Fix}\left(h_{i, s, t}\right) \cap U_{s} \times V_{t}$ is nonempty, and this fixed point set is a single point. By the splitting property we have $\iota\left(f^{\prime} \times g^{\prime}, U_{s} \times V_{t}\right)=\operatorname{ind}\left(h_{i, s, t}, U_{s} \times V_{t}\right)$. But for the same reasons, the splitting property of $\operatorname{ind}_{m n}$ gives $\operatorname{ind}_{m n}\left(f^{\prime} \times g^{\prime}, U_{s} \times V_{t}\right)=\operatorname{ind}\left(h_{i, s, t}, U_{s} \times V_{t}\right)$. Thus $\iota\left(f^{\prime} \times g^{\prime}, U_{s} \times V_{t}\right)=\operatorname{ind}_{m n}\left(f^{\prime} \times g^{\prime}, U_{s} \times V_{t}\right)$, and the above becomes:

$$
\iota(f \times g, U \times V)=\sum_{s=1}^{k} \sum_{t=1}^{l} \operatorname{ind}_{m n}\left(f^{\prime} \times g^{\prime}, U_{s} \times V_{t}\right) .
$$

Then the additivity and homotopy properties of ind $\mathrm{m}_{m n}$ give:

$$
\iota(f \times g, U \times V)=\operatorname{ind}_{m n}\left(f^{\prime} \times g^{\prime}, U \times V\right)=\operatorname{ind}_{m n}(f \times g, U \times V)
$$

as desired.

Using exactly the same proof as Theorem ??, we have the following solution property:

Theorem 13. [Solution property] If $\iota$ satisfies the additivity property of Theorem ?? and $\iota(f \times g, U \times V) \neq 0$, then $\operatorname{Fix}(f \times g) \cap(U \times V)$ is nonempty.

To prove our product formula we require a lemma about splittings of product maps. Let $\pi_{1}: X \times Y \rightarrow X$ and $\pi_{2}: X \times Y \rightarrow Y$ be the coordinate projections.

Lemma 14. Let $f: U \rightarrow D_{n}(X)$ be an n-valued map and $g: V \rightarrow D_{m}(Y)$ be an $m$-valued map. Then the product mn-valued map $f \times g$ splits if and only if each of $f$ and $g$ split. Specifically, if $\left\{f_{1}, \ldots, f_{n}\right\}$ and $\left\{g_{1}, \ldots, g_{m}\right\}$ are splittings of $f$ and $g$, then $f \times g$ splits as $\left\{f_{i} \times g_{j}\right\}$, where $i \in\{1, \ldots, n\}$ and $j \in\{1, \ldots, m\}$.

Proof. It is clear that if each of $f$ and $g$ split, say $f=\left\{f_{1}, \ldots, f_{n}\right\}$ and $g=$ $\left\{g_{1}, \ldots, g_{m}\right\}$, then $\left\{f_{i} \times g_{j}\right\}$ is a splitting of $f \times g$.

For the converse, assume that $f \times g$ splits, and we will show that each of $f$ and $g$ splits. Let $\left\{h_{1}, \ldots, h_{m n}\right\}$ be a splitting of $f \times g$. Because $f \times g$ is the product of $f$ and $g$ and $f$ is $n$-valued, the set of functions $\left\{\pi_{1} \circ h_{k} \mid k \in\{1, \ldots, m n\}\right\}$ has exactly $n$ elements, and it is clear that this is a splitting of $f$. Similarly the set of functions given by the various $\pi_{2} \circ h_{k}$ has exactly $m$ elements, and this is a splitting of $g$. 
Now we are ready to prove our product formula:

Theorem 15. Let $(f, U)$ and $(g, V)$ be admissible pairs. Then $(f \times g, U \times V)$ is an admissible pair, and

$$
\operatorname{ind}_{m n}(f \times g, U \times V)=\operatorname{ind}_{n}(f, U) \operatorname{ind}_{m}(g, V) .
$$

Proof. Let $\iota: \mathcal{P}_{n, m}(X, Y) \rightarrow \mathbb{R}$ be defined by $\iota(f \times g, U \times V)=\operatorname{ind}_{n}(f, U) \operatorname{ind}_{m}(g, V)$. It suffices to show that $\iota$ satisfies the three axioms of Theorem ??. In that case it must equal the fixed point index ind ${ }_{m n}$.

To show that $\iota$ satisfies the product homotopy axiom, let $(f, U)$ be admissibly homotopic to $\left(f^{\prime}, U\right)$ and $(g, V)$ be admissibly homotopic to $\left(g^{\prime}, V\right)$. Then by the homotopy property of the index we have:

$\iota(f \times g, U \times V)=\operatorname{ind}_{n}(f, U) \operatorname{ind}_{m}(g, V)=\operatorname{ind}_{n}\left(f^{\prime}, U\right) \operatorname{ind}_{m}\left(g^{\prime}, V\right)=\iota\left(f^{\prime} \times g^{\prime}, U \times V\right)$.

To show that $\iota$ satisfies the additivity axiom, let $\operatorname{Fix}(f \times g) \cap(U \times V) \subset\left(U_{1} \times\right.$ $\left.V_{1}\right) \sqcup\left(U_{2} \times V_{2}\right)$. Then we will have $\operatorname{Fix}(f) \cap U \subset U_{1} \sqcup U_{2}$ and $\operatorname{Fix}(g) \cap V \subset V_{1} \sqcup V_{2}$, and thus by the additivity property of the $\operatorname{ind}_{n}$ and $\operatorname{ind}_{m}$ we have:

$$
\begin{aligned}
\iota(f \times g, U \times V)= & \operatorname{ind}_{n}(f, U) \operatorname{ind}_{m}(g, V) \\
= & \left.\operatorname{ind}_{n}\left(f, U_{1}\right)+\operatorname{ind}_{n}\left(f, U_{2}\right)\right)\left(\operatorname{ind}_{m}\left(g, V_{1}\right)+\operatorname{ind}_{m}\left(g, V_{2}\right)\right) \\
= & \operatorname{ind}_{n}\left(f, U_{1}\right) \operatorname{ind}_{m}\left(g, U_{2}\right)+\operatorname{ind}_{n}\left(f, U_{2}\right) \operatorname{ind}_{m}\left(g, U_{1}\right) \\
& \quad+\operatorname{ind}_{n}\left(f, U_{2}\right) \operatorname{ind}_{m}\left(g, U_{1}\right)+\operatorname{ind}_{n}\left(f, U_{2}\right) \operatorname{ind}_{m}\left(g, U_{2}\right) \\
= & \iota\left(f \times g, U_{1} \times V_{1}\right)+\iota\left(f \times g, U_{2} \times V_{2}\right) \\
& \quad+\iota\left(f \times g, U_{1} \times V_{2}\right)+\iota\left(f \times g, U_{2} \times V_{1}\right)
\end{aligned}
$$

Since $U_{1}$ and $U_{2}$ are disjoint and $V_{1}$ and $V_{2}$ are disjoint and $\operatorname{Fix}(f \times g) \cap(U \times V) \subset$ $\left(U_{1} \times V_{1}\right) \sqcup\left(U_{2} \times V_{2}\right)$, the set $\operatorname{Fix}(f \times g) \cap\left(U_{1} \times V_{2}\right)$ is empty, and similarly $\operatorname{Fix}(f \times g) \cap\left(U_{2} \times V_{1}\right)$ is empty. Thus by Theorem ??, $\iota\left(f \times g, U_{1} \times V_{2}\right)$ and $\iota\left(f \times g, U_{2} \times V_{1}\right)$ are zero, and the above becomes

$$
\iota(f \times g, U \times V)=\iota\left(f \times g, U_{1} \times V_{1}\right)+\iota\left(f \times g, U_{2} \times V_{2}\right),
$$

and so $\iota$ satisfies the additivity axiom.

Finally we show that $\iota$ satisfies the splitting axiom. Let $f \times g$ split on $U \times V$. By Lemma ??, $f$ and $g$ split and the splitting of $f \times g$ has the form $\left(f_{i} \times g_{j}\right)$, where $\left(f_{i}\right)$ is a splitting of $f$ and $\left(g_{j}\right)$ is a splitting of $g$. To show that $\iota$ satisfies the splitting axiom, assume that $\operatorname{Fix}(f \times g) \cap U \times V=\operatorname{Fix}\left(f_{i} \times g_{j}\right) \cap U \times V$ is a single point. Then $\operatorname{Fix}\left(f_{i}\right) \cap U$ is a single point, and $\operatorname{Fix}\left(g_{j}\right) \cap V$ is a single point, and we have:

$\iota(f \times g, U \times V)=\operatorname{ind}_{n}(f, U) \operatorname{ind}_{m}(g, V)=\operatorname{ind}\left(f_{i}, U\right) \operatorname{ind}\left(g_{j}, V\right)=\operatorname{ind}\left(f_{i} \times g_{j}, U \times V\right)$

where the last equality is the product property of the fixed point index ind.

Taking $U$ and $V$ to be all of $X$ and $Y$ we obtain a product formula for the Lefschetz number: 
Theorem 16. Let $f: X \rightarrow D_{n}(X)$ be an n-valued map and $g: Y \rightarrow D_{m}(Y)$ be an m-valued map. Then

$$
L(f \times g)=L(f) L(g) .
$$

\section{A weaker set of axioms for manifolds}

In this section we prove a stronger version of Theorem ?? in the setting of $n$ valued maps on manifolds. In this case the additional structure on the space allows us to prove our uniqueness subject to a much weaker axiom in place of the splitting axiom. In particular our axioms for the index of an $n$-valued map will make no reference to the classical fixed point index.

We say an $n$-valued map $c: X \rightarrow D_{n}(X)$ is constant when there are points $c_{1}, \ldots, c_{n} \in X$ with $c(x)=\left\{c_{1}, \ldots, c_{n}\right\}$ for all $x \in X$. These points $c_{i}$ are called the values of $c$.

The following axiom mimics the axiom of the same name used in [?].

Axiom 17. (Normalization) Let $c: X \rightarrow D_{n}(X)$ be a constant $n$-valued map and $U \subset X$ an open set containing exactly one value of $c$. Then $\iota(c, U)=1$.

Our main result in this section uses the uniqueness theorem of [?] for the classical fixed point index on $\mathbb{R}^{d}$. The following is a special case of Theorem 4.2 of [?]:

Theorem 18. Let $\iota: \mathcal{C}\left(\mathbb{R}^{d}\right) \rightarrow \mathbb{R}$ be any function satisfying the following axioms:

- (Homotopy) Let $(f, U),(g, U) \in \mathcal{C}\left(\mathbb{R}^{d}\right)$ be admissibly homotopic. Then $\iota(f, U)=\iota(g, U)$.

- (Additivity) Let $(f, U) \in \mathcal{C}\left(\mathbb{R}^{d}\right)$ with $\operatorname{Fix}(f) \cap U \subseteq U_{1} \sqcup U_{2}$. Then $\iota(f, U)=$ $\iota\left(f, U_{1}\right)+\iota\left(f, U_{2}\right)$.

- (Normalization) Let $c$ be a constant map, then $\iota\left(c, \mathbb{R}^{d}\right)=1$.

Then $\iota$ is the local fixed point index.

We will use a classical fact about the fixed point index on manifolds. Because the index is a local topological invariant, it is preserved by the coordinate charts of the manifold. The following lemma follows from the classical definition of the index, or an axiomatic proof is given as part of the proof of Theorem 4.2 of [?].

Lemma 19. Let $X$ be a complex with $(f, U) \in \mathcal{C}(X)$, and assume that $U$ is homeomorphic to $\mathbb{R}^{d}$ for some dimension $d$ by a homeomorphism $\varphi: U \rightarrow \mathbb{R}^{d}$. Then the pair $\left(\varphi \circ f \circ \varphi^{-1}, \varphi(U)\right)$ is admissible in $\mathbb{R}^{d}$, and

$$
\operatorname{ind}(f, U)=\operatorname{ind}\left(\varphi \circ f \circ \varphi^{-1}, \varphi(U)\right) .
$$

Our main result for this section refers to the Homotopy and Additivity axioms from Section ?? (Axioms ?? and ??): 
Theorem 20. $X$ be a manifold. Then the local fixed point index $\operatorname{ind}_{n}$ is the unique real-valued function on $\mathcal{C}_{n}(X)$ satisfying the Homotopy, Additivity, and Normalization axioms.

Proof. Let $\iota: \mathcal{C}_{n}(X) \rightarrow \mathbb{R}$ satisfy the Homotopy, Additivity, and Normalization axioms. We will show that $\iota$ satisfies the Splitting Axiom (Axiom ??), and therefore by Theorem ?? it must be the fixed point index ind $_{n}$.

Let $(f, U) \in \mathcal{C}_{n}(X)$ such that $f$ splits on $U$ as $\left\{f_{1}, \ldots, f_{n}\right\}$, and let $\operatorname{Fix}(f) \cap$ $U=\operatorname{Fix}\left(f_{i}\right) \cap U$ be a single point $x \in U$. We will show that $\iota(f, U)=\operatorname{ind}\left(f_{i}, U\right)$. To simplify the notation let us assume that $i=1$.

By the excision property, and since $X$ is a manifold, we may assume that $U$ is a set homeomorphic to $\mathbb{R}^{d}$ where $d$ is the dimension of $X$. Furthermore, since $f(x)$ is a set of $n$ points and $U$ is a neighborhood of $x$, we may assume that $U$ is sufficiently small so that $f(U)$ consists of $n$ disjoint sets $\left\{f_{1}(U), \ldots, f_{n}(U)\right\}$, and $f_{j}(U) \cap U=\emptyset$ for $j \neq 1$.

Let $\varphi: U \rightarrow \mathbb{R}^{d}$ be a homeomorphism. Then for any selfmap $g: \mathbb{R}^{d} \rightarrow \mathbb{R}^{d}$, let $\bar{g}: U \rightarrow D_{n}(X)$ be the split $n$-valued map given by $\bar{g}=\left\{\varphi^{-1} \circ g \circ \varphi, f_{2}, \ldots, f_{n}\right\}$. This $\bar{g}$ is $n$-valued on $U$ since $\varphi^{-1} \circ g \circ \varphi(U) \subseteq U$ and $f_{j}(U)$ are each disjoint from $U$ and from one another for $j \neq 1$.

Let $\omega: \mathcal{C}\left(\mathbb{R}^{d}\right) \rightarrow \mathcal{C}_{n}(X)$ be defined by

$$
\omega(g, V)=\left(\bar{g}, \varphi^{-1}(V)\right) .
$$

Then $\iota \circ \omega$ is a real-valued function on $\mathcal{C}\left(\mathbb{R}^{d}\right)$. It is easy to see that $\iota \circ \omega$ satisfies the three axioms of Theorem ??, and so it is the fixed point index. But $(f, U)=\omega\left(\varphi \circ f_{1} \circ \varphi^{-1}, \varphi(U)\right)$, and so

$$
\iota(f, U)=\iota\left(\omega\left(\varphi \circ f_{1} \circ \varphi^{-1}, \varphi(U)\right)\right)=\operatorname{ind}\left(\varphi \circ f_{1} \circ \varphi^{-1}, \varphi(U)\right)=\operatorname{ind}\left(f_{1}, U\right)
$$

by Lemma ??. 